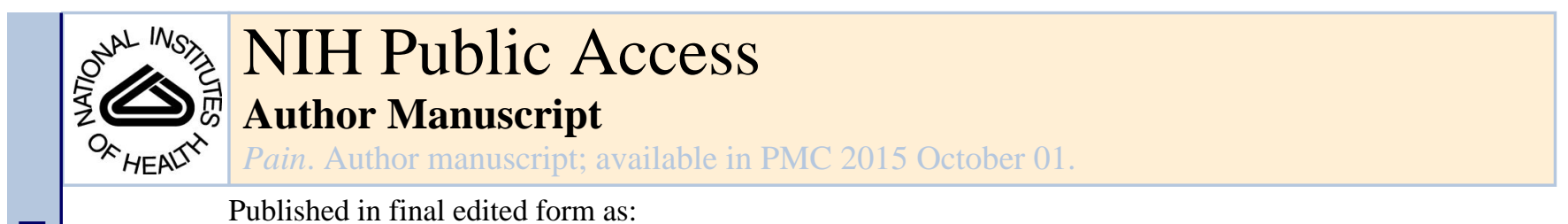

Published in final edited form as:

Pain. 2014 October ; 155(10): 2134-2143. doi:10.1016/j.pain.2014.08.007.

\title{
Pressure pain thresholds fluctuate with - but do not usefully predict - the clinical course of painful temporomandibular disorder
}

\author{
Gary D. Slade ${ }^{1,2,3}$, Anne E. Sanders ${ }^{1,2}$, Richard Ohrbach ${ }^{4}$, Roger B. Fillingim ${ }^{5}$, Ron \\ Dubner $^{6,7}$, Richard H. Gracely ${ }^{1,8}$, Eric Bair ${ }^{1,8,9}$, William Maixner ${ }^{1,8}$, and Joel D Greenspan 6,7
}

${ }^{1}$ Center for Pain Research and Innovation, University of North Carolina at Chapel Hill, Chapel Hill, NC ${ }^{2}$ Department of Dental Ecology, University of North Carolina at Chapel Hill, Chapel Hill, NC ${ }^{3}$ Department of Epidemiology, University of North Carolina at Chapel Hill, Chapel Hill, NC ${ }^{4}$ Department of Oral Diagnostic Sciences, University at Buffalo, Buffalo, NY ${ }^{5}$ Department of Community Dentistry \& Behavioral Science, University of Florida, Gainesville, FL ${ }^{6}$ Department of Neural and Pain Sciences, University of Maryland School of Dentistry, Baltimore, Maryland ${ }^{7}$ Brotman Facial Pain Center, University of Maryland School of Dentistry, Baltimore, Maryland ${ }^{8}$ Department of Endodontics, University of North Carolina at Chapel Hill, Chapel Hill, NC ${ }^{9}$ Department of Biostatistics, University of North Carolina at Chapel Hill, Chapel Hill, NC

\section{Abstract}

Central sensitization elicits pain hypersensitivity and is thought to be causally implicated in painful temporomandibular disorder (TMD). This causal inference is based on cross-sectional evidence that people with TMD have greater sensitivity than controls to noxious stimuli. We tested this inference in the OPPERA prospective cohort study of 3,258 adults with no lifetime history of TMD when enrolled (Visit 1). During five years of follow-up, one group labelled "persistent TMD cases" ( $\mathrm{n}=72)$ developed first-onset TMD by Visit 2 that persisted $\ 6$ months until Visit 3. Another group labelled "transient TMD cases" ( $n=75)$ developed first-onset TMD at Visit 2 which resolved by Visit 3. Randomly sampled "controls" ( $n=126)$ remained TMD-free throughout all three visits. At each visit, pressure pain thresholds (PPTs) were measured by algometry at 10 cranial and bodily sites. In persistent TMD cases, mean PPTs reduced $43 \mathrm{kPa}$ $(\mathrm{P}<0.0001)$ between Visits 1 and 2 and thereafter did not change significantly. In transient TMD cases, mean PPTs reduced $41 \mathrm{kPa}(\mathrm{P}<0.001)$ between Visits 1 and 2, and then increased $20 \mathrm{kPa}$ $(\mathrm{P}<0.001)$ by Visit 3 . These patterns were similar after excluding cranial sites symptomatic for

(C) 2014 Elsevier B.V. on behalf of International Association for the Study of Pain. All rights reserved.

Author for correspondence: Gary D Slade, Room 4501E, Koury Oral Health Sciences, UNC School of Dentistry, 385 South Columbia Street., CB\#7455, Chapel Hill, NC 27599-7455, USA. Phone 919-537-3273. FAX: 919-966-5339, gary_slade@ dentistry.unc.edu.

Conflict of interest statement: Drs. Slade, Fillingim, Gracely and Maixner are consultants with equity interests in Algynomics Inc., a company providing research services in personalized pain medication and diagnostics. All other authors declare no financial relationships that might represent a possible conflict of interest.

Publisher's Disclaimer: This is a PDF file of an unedited manuscript that has been accepted for publication. As a service to our customers we are providing this early version of the manuscript. The manuscript will undergo copyediting, typesetting, and review of the resulting proof before it is published in its final citable form. Please note that during the production process errors may be discovered which could affect the content, and all legal disclaimers that apply to the journal pertain. 
TMD. Importantly, Visit 1 PPTs had no clinically useful prognostic value in predicting first-onset TMD (odds ratio [OR] 1.07, $\mathrm{P}=0.15$ ). Among first-onset cases, Visit 2 PPTs were modest predictors of persistent TMD ( $\mathrm{OR}=1=.36, \mathrm{P}=0.002)$. In this longitudinal study, PPTs reduced when TMD developed then rebounded when TMD resolved. However, pre-morbid PPTs poorly predicted TMD incidence, countering the hypothesis that they signify mechanisms causing firstonset TMD.

\section{Keywords}

Temporomandibular disorder; epidemiology; pressure pain thresholds; algometry; longitudinal studies

\section{Introduction}

Pain thresholds vary markedly among people when blunt pressure is applied to their muscles and joints. Low pressure pain thresholds (PPTs) may signify a generalized state of pain sensitivity, variously attributed to altered sensory processing, dysregulated endocrine function, hyperinflammatory states, or psychological processes.[13] There is strong support for a role of central sensitization, defined as "amplification of neural signaling within the CNS that elicits pain hypersensitivity".[31] Central sensitization, in turn, is a putative cause of clinical pain syndromes including temporomandibular disorder (TMD).[31] Hence, pressure algometers, which provide a simple and non-invasive method to assess PPTs, are recommended for clinical care and research with TMD patients.[26]

Most evidence supporting a relationship between PPTs and clinical pain comes from studies using cross-sectional designs. [4; 5; 15; 19; 21; 22; 27] In one landmark case-control study, PPTs at facial locations were lower in patients with TMD than in TMD-free controls, irrespective of whether or not the location was symptomatic for TMD.[19] The investigators concluded that TMD was a consequence of general sensitization to otherwise normal sensations in the orofacial region and that centrally-mediated pain played a role "in either the onset or perpetuation of myogenous TMD".[19] Similar interpretations are reported in other cross-sectional studies of widespread pain.[13]

Fewer longitudinal studies have investigated PPTs and clinical pain, and their conclusions vary. In one study, pre-morbid PPTs were lower in people who later developed clinical shoulder pain than in people who did not.[14] In contrast, a prospective study of tensiontype headache found that pre-morbid PPTs did not predict its development.[2] Another study found that low PPTs did not predict development of widespread pain.[9] Prospective studies of people with acute whiplash injury report conflicting findings regarding the prognostic value of PPTs in predicting recovery. $[11 ; 30]$

Recently, we reported results from a prospective study of first-onset TMD that was part of the OPPERA project (Orofacial Pain: Prospective Evaluation and Risk Assessment).[7] Premorbid PPTs measured at enrollment were significant predictors of TMD incidence, although the effect was much weaker than the cross-sectional association between PPTS and chronic TMD in the OPPERA baseline case-control.[8] However, in the prospective study, 
we did not analyze changes in PPTs between enrollment and development of TMD, and we did not distinguish TMD that was transient from TMD that became persistent.

To address these gaps, the current study reports findings from PPTs measured in the OPPERA cohort before, during and after the development of clinical TMD. The first aim was to compare changes in PPTs between three groups: people who developed persistent TMD, people who developed transient TMD, and controls who remained free of TMD throughout the study. The second aim was to evaluate whether PPTs are useful clinically, either in predicting a person's risk of developing TMD or as a concurrent biomarker to discriminate between people who have TMD from people who do not. These aims do not consider potential interactions between PPTs and other risk factors for TMD which, instead, will be investigated in subsequent analysis of OPPERA data.

\section{Methods}

This paper reports findings from a nested case-control study of first-onset TMD that was part of the OPPERA project. Previous papers have described the relationship between this nested study and two other components of the OPPERA project: the baseline case-control study of chronic TMD [23] and the OPPERA prospective cohort study of first-onset TMD. [1] The following sections adopt STROBE guidelines [29] to elaborate on the nested study's design, setting, participants, variables, sample size justification and statistical analyses. All OPPERA study participants provided signed, informed consent to participate. The project was reviewed and approved by institutional review boards at each study site. Privacy of research participants is further protected by a Certificate of Confidentiality (NIDCR-06-17) between the National Institutes of Health and Dr. William Maixner, Program Director of OPPERA.

\section{Study design}

Nested case-control studies combine the efficiency of the case-control design with the strengths of longitudinal data from a prospective cohort study. Typically they collect additional new data from cohort members who develop the disease of interest (cases) and a subset of cohort members who remain disease-free (controls).[12] In the current study, cases were all participants in the OPPERA prospective cohort study who developed first-onset TMD and controls were an approximately equal number of participants who did not develop TMD.

\section{Study setting and enrollment screening criteria}

Between May 2006 and November 2009, the OPPERA prospective cohort study enrolled community-dwelling adults who had no significant history of TMD. Participants were recruited using advertisements, emails and flyers distributed in communities surrounding the four U.S. study sites: Baltimore, MD; Buffalo, NY; Chapel Hill, NC; and Gainesville, FL. Details of the study settings and study participants are reported elsewhere.[1] In summary, screening selection criteria were used to select volunteers who had all of the following characteristics: aged 18-44 years; English language fluency; intention to live in the area for at least two years; fewer than five headaches/month in the three months before enrollment; 
no history of significant TMD symptoms (that is, no orofacial pain in the month before enrollment and, prior to that period, no more than four days of orofacial pain per month); no prior diagnosis or treatment for TMD; and absence of 13 specific health conditions.

\section{Clinical examination to select TMD-free participants at enrollment (Visit 1)}

Volunteers who met screening selection criteria were asked to complete 15 questionnaires about psychological characteristics and health status [23] prior to their attendance at an OPPERA research clinic for a three-hour baseline assessment (Visit 1). The comprehensive examination protocol, adapted from the Research Diagnostic Criteria for TMD,[3] excluded participants found to have clinical TMD. Specifically, potential participants were excluded if they met either one of two criteria: 1) history of pain in the orofacial region for $\geq 5$ days in the prior 30 days; and 2) evoked pain in $\geq 3$ muscle locations (myalgia) or in $\geq 1$ temporomandibular joints (arthralgia). For the second criterion, study participants performed standardized jaw movements while examiners manually palpated ten anatomical locations: temporalis, masseter, lateral pterygoid and submandibular muscles and the temporomandibular joints (TMJs), each assessed bilaterally. Examiners were trained in the diagnostic criteria prior to study initiation and they had excellent inter-examiner reliability when compared to the study's reference examiner.[1] Using these procedures, 3,258 TMDfree subjects were enrolled into the prospective cohort study (Figure 1).

\section{Follow-up screening and Visit 2 examinations to select cases and controls for the nested study}

At three month intervals after enrollment, study participants completed a screening questionnaire that asked about TMD symptoms. Subjects reporting symptoms were invited to return to the respective research clinics for a follow-up assessment (Visit 2). Examiners followed the same comprehensive examination protocol to determine presence or absence of TMD using the criteria applied at Visit 1. In addition, participants with TMD completed questionnaires that included Gracely's ratio scales of facial pain unpleasantness and intensity during the preceding month.[6]

As each incident case was confirmed, one control was sampled at random from among the remaining symptom-free cohort members. Sampled controls were likewise invited to research clinics where examiners verified absence of clinical TMD. The median period between Visit 1 and Visit 2 examinations was 17 months (interquartile range $=10-26$ months). The sampling design matched cases and controls according to four criteria: 1) enrollment within 15 days of one another; 2) quarterly questionnaires completed within three months of one another; 3) same gender; and 4) enrollment at the same study site. The first two criteria created similar periods of follow-up for cases and controls, which is a requirement of the nested case-control design. Gender was a matching criterion because we expected greater TMD incidence in females than males. Study site matching was desirable to control for extraneous site-specific factors that are inherent in multi-site studies. Through May 2011, these procedures selected 456 participants for the nested case-control study including 235 participants who reported symptoms and were diagnosed with first-onset TMD and 221 matched controls (Figure 1). Among the matched controls, 25 had TMD when examined, resulting in 260 incident cases of first-onset TMD and 196 TMD-free 
controls. Excluded from this analysis were 159 Sub-clinical TMD participants who reported symptoms but who were negative for TMD when examined and 84 participants who reported symptoms but did not attend the examination (Figure 1).

\section{Follow-up clinical examination to determine TMD persistence at Visit 3}

Approximately six months after the Visit 2 examination, all participants in the nested casecontrol study were asked to return to research clinics for a third examination (Visit 3). The median period between Visit 2 and Visit 3 examinations was 8 months (interquartile range $=6-15$ months). The variability reflects our decision to make all possible efforts to reexamine participants in the nested case-control study, even when appointments had to be postponed or rescheduled. Of the 147 incident cases re-examined at Visit 3, 72 (49\%) had examiner-verified TMD and were labeled "persistent TMD" cases. We recognize that the duration of their TMD pain exceeds the six-month threshold used by the International Association for the Study of Pain[18] as the criterion to classify pain as chronic. However, we avoid the label "chronic" on the grounds that it is more appropriately classified using other dimensions in addition to duration[6] that were not assessed in this study. The remaining 75 participants (51\%) who no longer had examiner-verified TMD were labeled "transient TMD" case (Figure 1). Virtually all (126/127) of the controls who were reexamined at Visit 3 remained free of clinical TMD.

\section{Algometry measurements of pressure pain thresholds (PPTs)}

At each visit to research clinics, PPTs were measured using a pressure algometer. As described fully elsewhere,[8] bilateral measurements recorded at five anatomical locations (temporalis muscles, masseter muscles, TMJs, trapezius muscles, and the lateral epicondyles) yielded 10 measurements. Pressure was increased at the rate of $30 \mathrm{kPa}$ per second until either the participant indicated first pain sensation, or until $600 \mathrm{kPa}$ was applied. Measurements were repeated at each location either until two values were obtained within $20 \mathrm{kPa}$ of one another or until five trials were administered. The two closest values were then recorded.

\section{Variables used in this analysis}

For each study participant, repeated PPTs at left and right sides were averaged to produce five mean values, one for each pair of muscles or joints. For consistency with methods used in previous studies, these calculations included all threshold values ( $n=4,014$ values in 273 participants who attended all three visits). This included PPTs recorded at the cranial sites in which participants reported TMD-like pain that was evoked during clinical examination procedures. However, to create a measure of PPT that was not contaminated by TMD symptoms, we computed an additional outcome variable that excluded temporalis, masseter or TMJ measurements if pain was evoked at the same location during the clinical examination ( $n=3,073$ values in 273 participants). Hereafter, we refer to them as PPTs at non-symptomatic sites.

Demographic characteristics of gender, age, race and ethnicity were self-reported in screening interviews at the time of enrollment. Age was categorized into three groups for descriptive purposes and used as a continuous variable (in years) for statistical models. 
Questions about race and ethnicity were combined to form three groups: non-Hispanic White, African-American, and other or unstated racial-ethnic groups. For aim 2, each participant's interval between study visits was computed as the number of days between visits. Study site was a categorical variable denoting the study site at which participants were enrolled: FL, MD, NC, or NY.

\section{Statistical analysis}

For descriptive purposes, univariate statistics described the distribution of PPTs at each anatomical location and unadjusted means were compared among demographic groups. To test hypotheses for Aim 1, data were analyzed for the 273 participants who completed all three visits and adjusted means were computed using a general estimating equation (GEE) linear regression model. The model allowed for within-subject measurements that were repeated across visits and, within visits, at five anatomical locations. Explanatory variables in the model were study-group ( 3 categories: persistent TMD cases, transient TMD cases, and controls), visit (3 categories), study-group $\times$ visit interaction and four adjustment variables: study site (4 categories), age (continuous variable), gender ( 2 categories), raceethnicity (3 categories). The interaction term was included to allow the pattern of PPTs across visits to vary according to study group (that is, persistent TMD, transient TMD, and controls). Demographic variables were included in the models because the descriptive analysis revealed they were associated with PPTs. Study site was a covariate to control for extraneous factors that differ between sites in multi-site studies. We used GEE models in preference to repeated measures analysis of variance because GEE models use all available data, even for subjects who have missing data for one or more anatomical locations and/or visits. GEE models were created using the "genmod" procedure in SAS version 9.3, specifying an exchangeable working correlation matrix for within-subject measures. Adjusted means and standard errors were calculated using the "lsmeans" statement, while between-study-group and between-visit contrasts were calculated using the "Ismestimate" statement. When plotting means, reference values for chronic TMD cases were also displayed, based on values reported in the OPPERA baseline case-control study.[8]

The analysis for Aim 2 used mixed effect, unconditional binary logistic regression models to predict odds of clinical TMD, both at Visit 2 ( $\mathrm{n}=456$ participants with Visit 1 and Visit 2 data) and at Visit 3 ( $n=273$ participants with Visit 2 and Visit 3 data). The unconditional method was used, thereby ignoring the matched sampling design, because many pairs were incomplete due to non-examination of one pair member and because some participants selected as controls were found to be cases when examined (Figure 1). To aid in interpretation of prediction models, PPTs were transformed to negative z-scores. This was done separately for each anatomical location by subtracting the observed PPT from the sample mean for that anatomical location, and dividing the result by the standard deviation for that anatomical location. The resulting odds ratios therefore can be interpreted as the change in odds of TMD associated with a one standard deviation decrease in the PPT (that is, greater pain sensitivity). The fixed-effect, predictor variable was the z-transformed PPT, and there were five adjustment variables: interval between visits (continuous variable), study site (4 categories), age (continuous variable), gender ( 2 categories) and race-ethnicity (3 categories). Random effects were anatomical location nested within person. Odds ratios 
(ORs) and their 95\% confidence limits (95\% CLs) were calculated as measures of the strength of association between PPTs and odds of TMD. The ability of PPT to discriminate between cases and controls was indexed using the PPT's contribution to the area under the receiver operating characteristic curve (AUROC). To be "useful" for clinical diagnostic purposes, values of AUROC should exceed a threshold of 0.7, while values exceeding 0.9 are regarded as "excellent".[28] Mixed models were created using the "glimmix" procedure in SAS version 9.3, specifying a variance components covariance structure for the random effects. Mixed models were used because, in general, they are more appropriate for estimating subject-specific effects than GEE models, which are preferred for populationlevel effects.[10] We therefore used GEE models for Aim 1, where the focus was on mean changes in PPTs for the population from which study participants were selected, whereas mixed effect models were used for Aim2, which had a focus on predicting individuals' odds of clinical TMD.

Three different prognostic models were created: a) PPTs at visit 1 were used to predict odds of first-onset TMD at Visit 2; b) PPTs at visit 1 were used to predict odds of either persistent TMD (i.e., TMD classified both at Visit 2 and Visit 3) or transient TMD (i.e., TMD classified at Visit 2 and absence of TMD classified at Visit 3); and c) among participants with first-onset TMD, PPTs at Visit 2 were used to predict odds of persistent TMD at Visit 3 (i.e., TMD classified both at Visit 2 and Visit 3). Meanwhile, two models were created to evaluate associations with concurrent case-classification: d) PPTs at Visit 2 were used to predict odds of first-onset TMD at Visit 2; e) PPTs at Visit 3 were used to predict odds of persistent TMD at Visit 3.

Because the label "TMD" represents a cluster of clinical disorders that cause orofacial pain, we created sub-classifications of TMD to further investigate potential prognostic and concurrent associations of PPTs with specific types of TMD. One sub-classification distinguished between arthralgia alone, myalgia alone, and combined arthralgia + myalgia. Another sub-classification distinguished between TMD cases who reported low (below median) versus high (median or above) ratings of pain unpleasantness. A third subclassification distinguished between TMD cases who reported low versus high ratings of pain intensity.

We also explored the possibility that pain in other parts of the body might affect PPTs, thereby modifying observed relationships between PPTs and TMD. Responses to baseline questionnaires about health status were used to classify four pain-related conditions: a) the number of different types of headache during the preceding year; b) low back pain during the preceding year; c) irritable bowel syndrome; and d) a checklist of 20 comorbid conditions related to pain, ranging from arthritis to sleep apnea. The four were chosen because all were strong predictors of TMD in the OPPERA baseline case-control study [18] and the OPPERA prospective cohort study of TMD incidence.[20]

For both aims, the threshold for statistical significance was $\mathrm{P}<0.05$ based on two-tailed tests. Inferences about clinical significance were based on the magnitude of mean differences (Aim 1) and effect measures (odds ratios and AUROC). 


\section{Sample size justification}

In the OPPERA prospective cohort study the target sample size was 3,200 enrollees because that number was expected to yield 196 cases of first-onset TMD during a three-year followup period. Calculations made when designing the study indicated that those numbers would provide $80 \%$ statistical power to detect risk ratios of at least 1.8 for risk predictors with as few as $15 \%$ of people in the high-risk category.[1] Post-hoc calculations were made for the 454 participants in this nested case-control study who were examined at Visit 2, revealing $80 \%$ power to detect differences in PPTs between cases and controls that were as small as 0.27 standard deviations. For the 273 participants in the nested case-control study seen at all three visits, the corresponding minimum difference was 0.35 standard deviations.

\section{Results}

Upon enrollment in the OPPERA prospective cohort study, the 3,258 TMD-free study participants ranged in age from 18 to 44 years (mean=27 years), 57\% were female and $50 \%$ were non-Hispanic Whites. Through May, 2011, 478 enrollees reported TMD symptoms in at least one follow-up questionnaire, 2,259 reported no symptoms, and 521 enrollees completed no follow-up questionnaires (Figure 1). Examinations at Visit 2 identified 456 participants eligible for the nested case-control study (260 first-onset cases and 196 TMDfree controls). However, 182 (113 cases and 69 controls) did not attend the Visit 3 examination and one control at Visit 2 who developed TMD at Visit 3 was excluded. Thus 273 participants in the nested case-control study had data from all three visits. Excluded from the analysis were 159 "Subclinical TMD" particpiants who reported symptoms but who were found by examiners not to have TMD at Visit 2.

In most instances, mean PPTs in groups lost to follow-up were similar to the larger group from which they originated.(Appendix Figure 1) For example, mean PPTs at cranial sites for the 521 participants who completed no follow-up questionnaires were within $5 \mathrm{kPa}$ of means for all enrollees at Visit 1. When larger differences were found, they tended to be PPTs from bodily sites. For example, the aforementioned groups differed by $16 \mathrm{kPa}$ in mean trapezius PPTs, and by $19 \mathrm{kPa}$ in mean lateral epicondyle PPTs. Previous analysis of demographic patterns in cohort retention showed that loss to follow-up was greater among males than females, and among African Americans compared to other racial-ethnic groups.[1]

Among all 456 participants who at least completed Visits 1 and 2, mean PPTs at Visit 1 varied significantly according to age, gender and race-ethnicity for at least one anatomical location (Table 1). The largest variation was between genders, where the lateral epicondyle PPT was $100 \mathrm{kPa}(23 \%)$ lower in females than in males. Females also had significantly lower PPTs at all other anatomical sites. The general similarity of demographic associations, regardless of anatomical location, was consistent with strong correlations observed between all pairs of PPTs (Appendix Table 1).

In the analysis of PPTs from all five anatomical sites in participants who completed all three visits, the three study groups displayed distinctive temporal patterns in mean PPTs (Figure 2A). Differences in temporal patterns among groups were evidenced by a statistically significant $(\mathrm{P}<0.001)$ interaction between study group and visit in the GEE model used to 
calculate adjusted means. For those who became persistent cases, the mean PPT of $256 \mathrm{kPa}$ at enrollment reduced by $43 \mathrm{kPa}(17 \%$; $\mathrm{P}<0.001)$ at the time of first-onset TMD, and increased by only $9 \mathrm{kPa}(4 \% ; \mathrm{P}=0.056)$ by Visit 3 . For those who developed transient TMD, the mean PPT of $270 \mathrm{kPa}$ at enrollment reduced by $41 \mathrm{kPa}(15 \% ; \mathrm{P}<0.001)$ at the time of first-onset TMD, but increased by $20 \mathrm{kPa}(9 \%, \mathrm{P}<0.001)$ at Visit 3, when the TMD had remitted. Among controls, differences between visits were statistically significant, though small in magnitude, varying by no more than $10 \mathrm{kPa}(4 \%)$ between visits. The patterns were strikingly similar when data were restricted to PPTs recorded at non-symptomatic sites (Figure 2B).

Table 2 quantifies the changes depicted in Figure 2 and includes additional contrasts in PPTs between study groups. Of note, Visit 1 PPTs were $20 \mathrm{kPa}$ lower (8\%, P=0.003) in participants who became persistent cases compared to Visit 1 PPTs in controls. However, Visit 1 PPTs in participants who became transient cases were only $2 \mathrm{kPa}$ lower than Visit 1 PPTs in controls $(\mathrm{P}=0.747)$. Although not included in Table 2, participants with Subclinical TMD at Visit 2 had PPT values at Visit 2 (mean $=245.8$, standard error $=7.9, \mathrm{n}=159$ participants) that that were intermediate between controls and the two groups of TMD cases at the same visit (Table 2). For the Subclinical TMD participants, this represented a 7\% reduction $(\mathrm{P}<0.001)$ of their Visit 1 PPTs (mean= 263.8, standard error=7.8).

The temporal pattern was similar using mean PPTs for each of the five anatomical locations (Appendix Fig 2A-2E). In general, PPTs reduced considerably at the time of first-onset TMD and for persistent cases, PPTs did not change significantly thereafter. In contrast, among transient cases, PPTs increased upon remission of TMD. One exception to this general pattern was seen at the lateral epicondyle (Appendix Fig 2E) where PPTs did not increase between Visits 2 and 3 in transient cases. However, temporal patterns among study groups did not differ to a statistically significant degree across body sites, as evidenced by a separate GEE model that added a three way interaction of location $\times$ study group $\times$ visit (for the interaction's score statistic, Chi-square $=19.7$, degrees of freedom $=16, \mathrm{P}=0.2339$ ).

Using data from all anatomical sites, pre-morbid (i.e., Visit 1) PPTs were, at best, weak predictors of TMD incidence (Table 3). For the 456 participants with at least two visits, Visit 1 PPTs were not significantly associated with odds of first-onset TMD at Visit 2 $(\mathrm{OR}=1.07,95 \% \mathrm{CL}=0.98,1.17)$ and were no better than chance (AUROC $=0.50)$ at discriminating between incident cases and controls. Visit 1 PPTs were statistically significant, but modest, predictors of persistent $\mathrm{TMD}$ at Visit 3, $(\mathrm{OR}=1.18,95 \% \mathrm{CL}=1.00$, 1.38 ) and discrimination was poor (AUROC $=0.53$ ). However, among participants who developed first-onset TMD, Visit 2 PPTs were statistically significant predictors of persistent TMD at Visit 3, although the effect was only of modest clinical significance $(\mathrm{OR}=1.36,95 \% \mathrm{CL}=1.11,1.66 ; \mathrm{AUROC}=0.54)$. Meanwhile, there were statistically-and clinically-significant concurrent associations between PPTs and TMD case-classification (Table 4). PPTs measured at Visit 2 had a moderately strong concurrent association with first-onset TMD at Visit $2(\mathrm{OR}=2.62,95 \% \mathrm{CL}=2.32,2.95$; $\mathrm{AUROC}=0.66)$. The concurrent association between Visit 3 PPTs and TMD case-classification at Visit 3 was also statistically significant although not as strong $(\mathrm{OR}=1.93,95 \% \mathrm{CL}=1.62,2.30$; AUROC $=0.61$ ). 
Analysis of TMD sub-classifications did not appreciably alter the findings. Visit 1 PPTs were weak and statistically-non-significant predictors of all three anatomical subclassifications at Visit 2: myalgia alone ( $n=62$ TMD cases), arthralgia alone ( $n=10$ TMD cases) and mixed arthralgia+myalgia ( $\mathrm{n}=188$ TMD cases; Model 1, Appendix Table 2). However, Visit 2 PPTs were strongly associated with each of the anatomical subclassifications at Visit 2 (Model 2, Appendix Table 2). Likewise, Visit 1 PPTs were equally weak predictors of TMD with high pain unpleasantness and TMD with low pain unpleasantness (Model 3, Appendix Table 2). The same was true when TMD was subclassified according to pain intensity (Model 4, Appendix Table 2). However, for the concurrent associations at Visit 2 PPTs, low thresholds were more strongly associated with the sub-types that had more severe symptoms at Visit 2 (Models 5 and 6, Appendix Table 2)

While considerable numbers of study participants at enrollment reported headache, low back pain, irritable bowel syndrome, or comorbid pain-related conditions, PPTs at Visit 1 were not systematically associated with those conditions (Appendix Table 3). Mean PPTs at four anatomical sites varied according to the number of different types of headaches, with nominal P-values ranging from 0.010 to 0.048 . However there was no consistent direction in the relationship: PPTs were greatest among participants with no headaches or with $\geq 4$ types of headache, whereas PPTs were lower for participants with intermediate numbers of headache types. Other conditions were associated with mean PPTs in expected directions, but P-values ranged from 0.082 to 0.750 . Furthermore, none of the P-values in Appendix Table 3 achieved the threshold of statistical significance after adjusting for the 20 tests. Overall, the findings suggested that previously noted associations between PPTs and TMD were unlikely to be altered by these pain-related conditions.

\section{Discussion}

In this community-based, longitudinal study of U.S. adults, PPTs fluctuated in synchrony with the course of painful TMD. Compared to pre-morbid values recorded at enrollment, PPTs decreased significantly at the time of first-onset TMD ascertainment. PPTs remained lowered in the group whose clinical TMD persisted. However in those whose TMD pain resolved, PPTs increased to near pre-morbid levels. When viewed cross-sectionally, we found moderately strong, concurrent associations between TMD case-classification and PPTs determined at the same visit. However, the novel contribution of this study is that premorbid PPTs had no clinically-useful prognostic value in predicting who would and who would not develop first-onset TMD. Specifically, AUROC values were well below the benchmark of 0.7,[28] meaning that, for a given PPT value, the true-positive proportion of TMD cases was not much greater than the false-positive proportion. At best, PPTs at the time of first-onset TMD were statistically significant, though modest, predictors of TMD becoming persistent. The general lack of prognostic utility challenges the prevailing hypothesis that pre-morbid sensitivity to pressure pain is indicative of sensitized pain pathways that contribute causally to the risk of developing painful TMD. This conclusion assumes that complex pain conditions have multiple causes, none of which are necessary or sufficient. Instead, we adopt the epidemiologic concept of a cause as something which, when blocked or removed, results in less disease within a population.[20] 
Loss to follow-up is a concern in all longitudinal studies, particularly when it is associated with characteristics used to test study hypotheses. In this study, most PPT measurements at Visit 1 were not associated with loss to follow-up, although lateral epicondyle PPTs tended to be lower in people lost to follow-up. Meanwhile, PPT changes between visits in the persistent TMD group appeared different at the lateral epicondyle compared to other anatomical locations. While this finding might suggest anatomical specificity in the relationship between PPTs and TMD, we are reluctant to endorse the idea because of potential loss to follow-up bias, and because the three-way interaction of location $\times$ study group $\times$ visit was not statistically significant.

Different patterns of TMD may have been observed had study participants been followed for longer periods than the median 17 months between Visits 1 and 2, or the median 8 months between Visits 2 and 3. Another limitation was that PPTs were measured only at clinic visits, not continually throughout the study. Clinic visits were scheduled as soon as possible after symptoms were reported, although in practice, there was a median delay of 14 days.[1] PPTs therefore could have reduced before being measured at Visit 2, and possibly even before TMD developed. If the latter occurred, the true prognostic value of PPT measurements would be underestimated in this study. However, continual monitoring of PPTs at home is not feasible. Furthermore, if reductions in PPTs were informative only within a window of a few weeks, the information would have little utility in most clinical settings. For these reasons, we believe that current study provides a realistic testing ground and justifies our overall conclusion that pre-morbid PPT measurements are not useful clinical predictors of first-onset TMD.

This analysis was limited to PPTs because our previous analyses found them to be strongly associated with clinical TMD. [7; 8] In contrast, temporal summation of mechanical cutaneous "pinprick" stimuli was only weakly associated with chronic TMD in the baseline case-control analysis[8] and it did not predict incidence of TMD.[7] Meanwhile, temporal summation of thermal stimuli was not associated with chronic TMD [8] and was only weakly associated with TMD incidence.[7] Both findings were surprising because temporal summation is a more specific indicator than PPTs of central sensitization. However, effects of temporal summation on clinical pain appear to be complex: we have since re-analyzed the baseline case-control study and found that associations between temporal summation and TMD were dependent on initial sensitivity to thermal pain. In this paper, we therefore focused on PPTs, expecting them to have greatest prognostic value for changes in clinical TMD.

While the overall weak prognostic value of pre-morbid PPTs was unexpected, the finding has precedents in studies of other idiopathic pain syndromes. In a community-based study of 674 Danes followed for 12 years, 63 developed headache, but PPTs measured at enrollment did not predict risk of developing headaches[2]. However, PPTs decreased at follow-up in subjects who developed chronic tension-type headache over the 12-year period, a result that mirrors the changes seen here in people who developed first-onset TMD. In another community-based study, 170 Danish adults were followed for 8 years and 57 developed low back pain, although baseline PPTs did not predict risk of its development.[17] In a community-based study of 228 U.K. adults, PPTs measured at enrollment were not 
significant predictors of widespread pain development.[9] However, other studies have found that PPTs can predict clinical pain. A longitudinal study of 12 newly-employed female process workers found that PPTs at enrollment were significantly lower for the six who developed shoulder pain six months later compared to the six who did not.[14] A study of 35 diabetic patients undergoing lower limb amputation found that pre-operative PPTs measured at the limb to be amputated were weak predictors of post-operative pain intensity after one week but did not predict pain after six months.[16] Overall, while the findings from small, selected study samples indicate that low PPTs can have modest, short-term prognostic value, results from larger community-based studies suggest that they have little prognostic value in the population at large.

In the current study and in the longitudinal studies noted above, the distinction between firstonset pain and persistent pain was based solely on the duration of clinical pain. While time is the one criterion used in some taxonomies to define chronic pain,[18] it arguably is not sufficient. If, in addition, chronic pain is "perpetuated by factors that are both pathogenically and physically remote from the originating cause",[6] it is likely that some of the persistent TMD cases in this study would not qualify as cases of chronic pain. This is supported by the observation that mean PPTs in persistent cases were never as low as the mean PPTs in chronic TMD cases from the OPPERA baseline case-control study. Of note, these persistent cases had experienced TMD for up to a year, whereas most of the chronic cases reported TMD of at least three years duration.[23] It follows that low-premorbid PPTs might yet prove to be useful clinical predictors for a subgroup of these persistent cases who develop truly chronic pain. To investigate that possibility, future studies of longer duration will need to evaluate a broader set of pathogenic factors contributing to this complex pain condition.

The current study has three major strengths. Firstly, the sample size of 260 incident cases of first-onset TMD far exceeds numbers of incident cases reported in other longitudinal studies noted above. Secondly we had analytic flexibility to select only anatomical locations that were non- symptomatic for TMD. This revealed that pressure pain sensitivity was not merely a consequence of TMD symptoms, but was generalized to other anatomical locations. The third major strength was measurement of PPTs at three points in time. This gave rise to the novel finding that low PPTs at the time of first-onset TMD were modestly predictive of persistent TMD. Although the corresponding AUROC value was well below the designated benchmark for "useful" clinical prediction,[28] the result suggests that low PPTs may offer some etiologic insight into the transition from acute to chronic pain. We caution, however, that this possibility needs to be reconciled with the weaker effect of premorbid PPTs in predicting persistent TMD. One possibility is that low PPTs in an asymptomatic person signifies a trait predisposing the person to alterations in central nociceptive pathways. In someone with this predisposition, the first-onset of painful TMD then serves as a trigger to increase synaptic efficacy of neurons in central nociceptive pathways which is the hallmark of central sensitization.[31] The idea that clinical pain might be a trigger is supported by findings from an experimental study of 28 women with fibromyalgia showing that peripheral mechanisms elicited central sensitization at the site of injury.[25] This proposed combination of a predisposing trait and a triggering event is a more nuanced interpretation than the general idea that central sensitization contributes to clinical pain syndromes such as TMD. It adds support for the interpretation of Reid et al [19] 
that central mechanisms are involved in perpetuation of TMD, although it refutes their suggestion that central mechanisms contribute to its onset.

In summary, while pre-morbid algometry measurements of PPTs were not useful in predicting the clinical course of TMD, the measurements appear to provide insight into mechanisms causing general sensitization in people with recent onset-TMD. That insight should help clinicians and their patients understand that TMD pain symptoms can have consequences that spread beyond the orofacial region.

\section{Supplementary Material}

Refer to Web version on PubMed Central for supplementary material.

\section{Acknowledgments}

This work was supported by the National Institutes of Health, and National Institutes of Dental and Cranial Research (grant numbers U01DE17018 and R03-DE022595). The OPPERA program also acknowledges resources specifically provided for this project by the participating institutions: Battelle Memorial Institute; University at Buffalo; University of Florida; University of Maryland; University of North Carolina at Chapel Hill.

\section{References}

1. Bair E, Brownstein NC, Ohrbach R, Greenspan JD, Dubner R, Fillingim RB, Maixner W, Smith SB, Diatchenko L, Gonzalez Y, Gordon SM, Lim PF, Ribeiro-Dasilva M, Dampier D, Knott C, Slade GD. Study Protocol, Sample Characteristics, and Loss to Follow-Up: The OPPERA Prospective Cohort Study. The journal of pain : official journal of the American Pain Society. 2013; 14(12 Suppl):T2-T19. [PubMed: 24275220]

2. Buchgreitz L, Lyngberg AC, Bendtsen L, Jensen R. Increased pain sensitivity is not a risk factor but a consequence of frequent headache: a population-based follow-up study. Pain. 2008; 137(3):623630. [PubMed: 18061350]

3. Dworkin S, LeResche L. Research diagnostic criteria for temporomandibular disorders: review, criteria, examinations and specifications, critique. J Craniomandib Disord. 1992; 6(4):301-355. [PubMed: 1298767]

4. Farella M, Michelotti A, Steenks MH, Romeo R, Cimino R, Bosman F. The diagnostic value of pressure algometry in myofascial pain of the jaw muscles. Journal of oral rehabilitation. 2000; 27(1):9-14. [PubMed: 10632838]

5. Fernandez-de-las-Penas C, Galan-del-Rio F, Fernandez-Carnero J, Pesquera J, Arendt-Nielsen L, Svensson P. Bilateral widespread mechanical pain sensitivity in women with myofascial temporomandibular disorder: evidence of impairment in central nociceptive processing. J Pain. 2009; 10(11):1170-1178. [PubMed: 19592309]

6. Gracely RH, McGrath F, Dubner R. Ratio scales of sensory and affective verbal pain descriptors. Pain. 1978; 5(1):5-18. [PubMed: 673440]

7. Greenspan JD, Slade GD, Bair E, Dubner R, Fillingim RB, Ohrbach R, Knott C, Diatchenko L, Liu Q, Maixner W. Pain Sensitivity and Autonomic Factors Associated With Development of TMD: The OPPERA Prospective Cohort Study. The journal of pain : official journal of the American Pain Society. 2013; 14(12 Suppl):T63-T74 e66. [PubMed: 24275224]

8. Greenspan JD, Slade GD, Bair E, Dubner R, Fillingim RB, Ohrbach R, Knott C, Mulkey F, Rothwell R, Maixner W. Pain sensitivity risk factors for chronic TMD: descriptive data and empirically identified domains from the OPPERA case control study. The journal of pain : official journal of the American Pain Society. 2011; 12(11 Suppl):T61-74. [PubMed: 22074753]

9. Gupta A, McBeth J, Macfarlane GJ, Morriss R, Dickens C, Ray D, Chiu YH, Silman AJ. Pressure pain thresholds and tender point counts as predictors of new chronic widespread pain in somatising subjects. Ann Rheum Dis. 2007; 66(4):517-521. [PubMed: 17012291] 
10. Hu FB, Goldberg J, Hedeker D, Flay BR, Pentz MA. Comparison of population-averaged and subject-specific approaches for analyzing repeated binary outcomes. Am J Epidemiol. 1998; 147(7):694-703. [PubMed: 9554609]

11. Kasch H, Stengaard-Pedersen K, Arendt-Nielsen L, Staehelin Jensen T. Pain thresholds and tenderness in neck and head following acute whiplash injury: a prospective study. Cephalalgia. 2001; 21(3):189-197. [PubMed: 11442553]

12. Last, JM.; Spasoff, RA.; Harris, SS.; Thuriaux, MC. International Epidemiological Association. A dictionary of epidemiology. Oxford; New York: Oxford University Press; 2001.

13. Lautenbacher S, Rollman GB, McCain GA. Multi-method assessment of experimental and clinical pain in patients with fibromyalgia. Pain. 1994; 59(1):45-53. [PubMed: 7854801]

14. Madeleine P, Lundager B, Voigt M, Arendt-Nielsen L. The effects of neck-shoulder pain development on sensory-motor interactions among female workers in the poultry and fish industries. A prospective study. Int Arch Occup Environ Health. 2003; 76(1):39-49. [PubMed: 12592581]

15. Malow RM, Grimm L, Olson RE. Differences in pain perception between myofascial pain dysfunction patients and normal subjects: a signal detection analysis. J Psychosom Res. 1980; 24(6):303-309. [PubMed: 7205718]

16. Nikolajsen L, Ilkjaer S, Jensen TS. Relationship between mechanical sensitivity and postamputation pain: a prospective study. European journal of pain. 2000; 4(4):327-334. [PubMed: 11124004]

17. O'Neill S, Kjaer P, Graven-Nielsen T, Manniche C, Arendt-Nielsen L. Low pressure pain thresholds are associated with, but does not predispose for, low back pain. Eur Spine J. 2011; 20(12):2120-2125. [PubMed: 21512842]

18. Ohrbach R, Fillingim RB, Mulkey F, Gonzalez Y, Gordon S, Gremillion H, Lim PF, RibeiroDasilva M, Greenspan JD, Knott C, Maixner W, Slade G. Clinical findings and pain symptoms as potential risk factors for chronic TMD: descriptive data and empirically identified domains from the OPPERA case-control study. J Pain. 2011; 12(11 Suppl):T27-45. [PubMed: 22074750]

19. Reid KI, Gracely RH, Dubner RA. The influence of time, facial side, and location on pain-pressure thresholds in chronic myogenous temporomandibular disorder. J Orofac Pain. 1994; 8(3):258-265. [PubMed: 7812223]

20. Sanders AE, Slade GD, Bair E, Fillingim RB, Knott C, Dubner R, Greenspan JD, Maixner W, Ohrbach R. General health status and incidence of first-onset temporomandibular disorder: the OPPERA prospective cohort study. The journal of pain : official journal of the American Pain Society. 2013; 14(12 Suppl):T51-62. [PubMed: 24275223]

21. Santos Silva RS, Conti PC, Lauris JR, da Silva RO, Pegoraro LF. Pressure pain threshold in the detection of masticatory myofascial pain: an algometer-based study. J Orofac Pain. 2005; 19(4): 318-324. [PubMed: 16279483]

22. Sarlani E, Grace EG, Reynolds MA, Greenspan JD. Evidence for up-regulated central nociceptive processing in patients with masticatory myofascial pain. J Orofac Pain. 2004; 18(1):41-55. [PubMed: 15029872]

23. Slade GD, Bair E, By K, Mulkey F, Baraian C, Rothwell R, Reynolds M, Miller V, Gonzalez Y, Gordon S, Ribeiro-Dasilva M, Lim PF, Greenspan JD, Dubner R, Fillingim RB, Diatchenko L, Maixner W, Dampier D, Knott C, Ohrbach R. Study methods, recruitment, sociodemographic findings, and demographic representativeness in the OPPERA study. The journal of pain : official journal of the American Pain Society. 2011; 12(11 Suppl):T12-26. [PubMed: 22074749]

24. Slade GD, Fillingim RB, Sanders AE, Bair E, Greenspan JD, Ohrbach R, Dubner R, Diatchenko L, Smith SB, Knott C, Maixner W. Summary of findings from the OPPERA prospective cohort study of incidence of first-onset temporomandibular disorder: implications and future directions. The journal of pain : official journal of the American Pain Society. 2013; 14(12 Suppl):T116-124. [PubMed: 24275219]

25. Staud R, Nagel S, Robinson ME, Price DD. Enhanced central pain processing of fibromyalgia patients is maintained by muscle afferent input: a randomized, double-blind, placebo-controlled study. Pain. 2009; 145(1-2):96-104. [PubMed: 19540671] 
26. Svensson P, Baad-Hansen L, Pigg M, List T, Eliav E, Ettlin D, Michelotti A, Tsukiyama Y, Matsuka Y, Jaaskelainen SK, Essick G, Greenspan JD, Drangsholt M. Guidelines and recommendations for assessment of somatosensory function in oro-facial pain conditions--a taskforce report. Journal of oral rehabilitation. 2011; 38(5):366-394. [PubMed: 21241350]

27. Svensson P, List T, Hector G. Analysis of stimulus-evoked pain in patients with myofascial temporomandibular pain disorders. Pain. 2001; 92(3):399-409. [PubMed: 11376913]

28. Swets JA. Measuring the accuracy of diagnostic systems. Science. 1988; 240(4857):1285-1293. [PubMed: 3287615]

29. von Elm E, Altman DG, Egger M, Pocock SJ, Gotzsche PC, Vandenbroucke JP. The Strengthening the Reporting of Observational Studies in Epidemiology (STROBE) statement: guidelines for reporting observational studies. J Clin Epidemiol. 2008; 61(4):344-349. [PubMed: 18313558]

30. Walton DM, Macdermid JC, Nielson W, Teasell RW, Reese H, Levesque L. Pressure pain threshold testing demonstrates predictive ability in people with acute whiplash. J Orthop Sports Phys Ther. 2011; 41(9):658-665. [PubMed: 21885908]

31. Woolf CJ. Central sensitization: implications for the diagnosis and treatment of pain. Pain. 2011; 152(3 Suppl):S2-15. [PubMed: 20961685] 


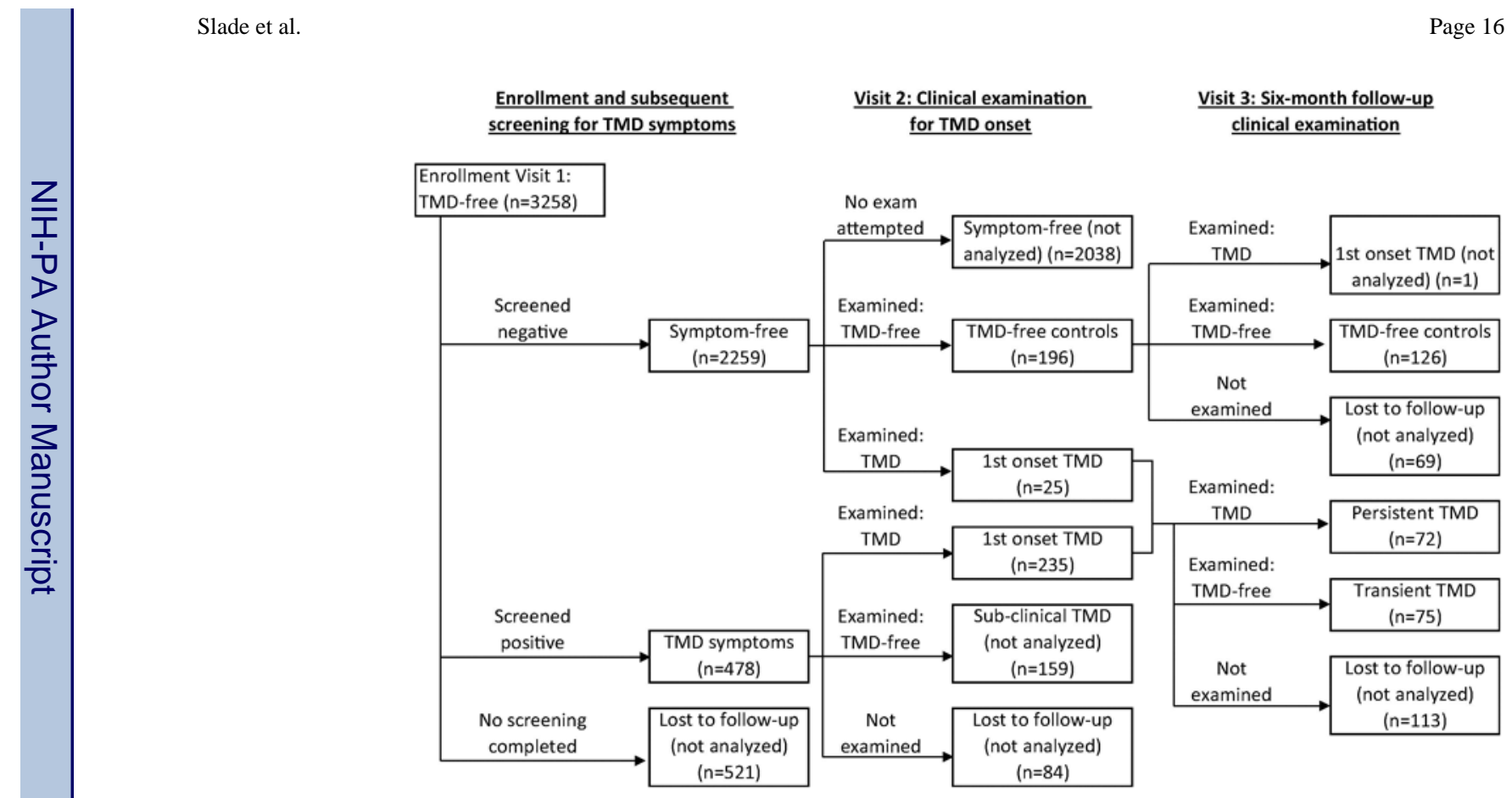

Figure 1. Flowchart of enrollment and follow-up in the OPPERA nested case-control study of TMD incidence 

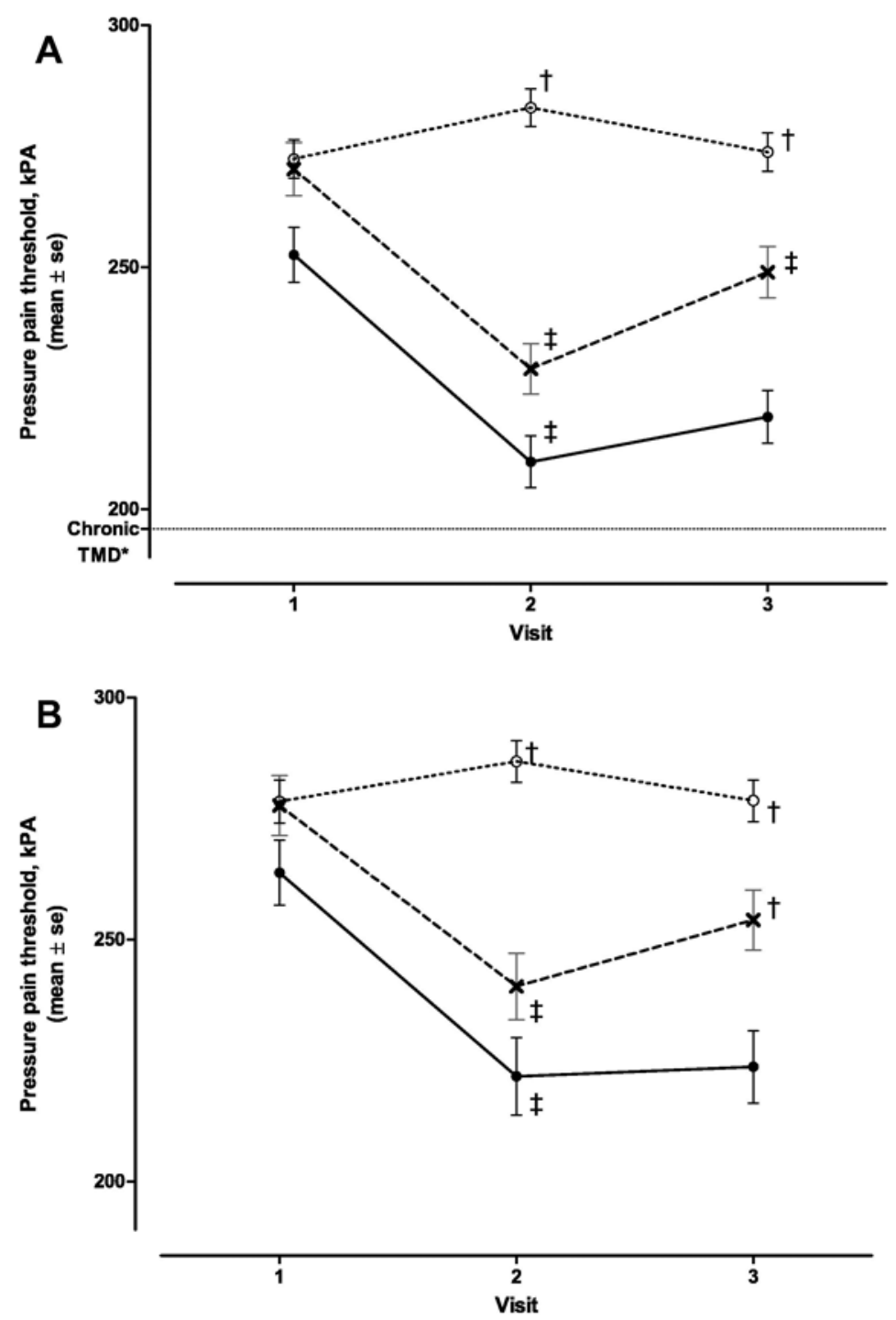

Figure 2.

Adjusted mean pressure pain thresholds pooled from five anatomical locations measured at three visits in each of three study groups: OPPERA nested case-control study of TMD incidence. Data are from 72 persistent TMD cases who developed first-onset TMD at Visit 2 that persisted at Visit $3(\bigcirc), 75$ transient TMD cases who developed first-onset TMD at visit 2 that remitted at Visit $3(\times)$ and 126 TMD-free controls $(\bigcirc)$. Adjusted means were computed using a generalized estimating equation for within-subject measurements that were repeated across visits and, within visits, across five anatomical sites. Covariates in the model were: study-group (persistent TMD, transient TMD or control), visit (1, 2 or 3), study-group $\times$ visit interaction and five adjustment variables: age (continuous variable), gender (2 groups), race-ethnicity ( 3 groups), study site (4 groups) and anatomical location (5 groups). 2A shows adjusted means from all 4014 pressure pain thresholds while $2 \mathrm{~B}$ shows adjusted means from 3,073 pressure pain thresholds that excluded measurements made at cranial sites in which participants reported TMD-like pain during clinical examination. Symbols represent $\mathrm{P}$-values testing the null hypothesis that the adjusted mean threshold at 
one visit is equal to the adjusted mean threshold at the preceding visit in the same study group: $\ddagger \mathrm{P}<0.01 ; \dagger 0.01<\mathrm{P}<0.05$. * Reference values for chronic TMD are from Greenspan et al, 2011. [8] 


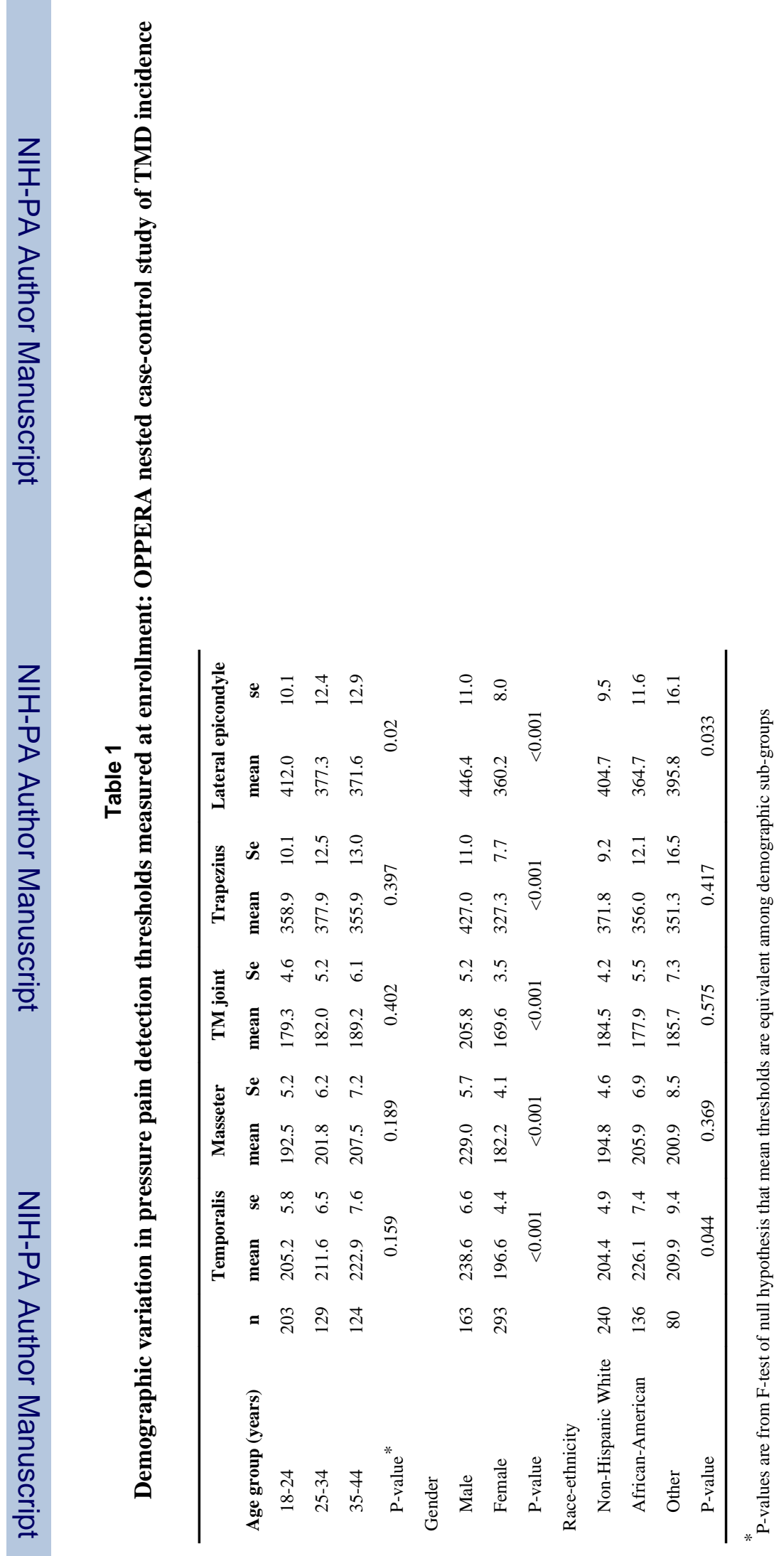

Pain. Author manuscript; available in PMC 2015 October 01. 


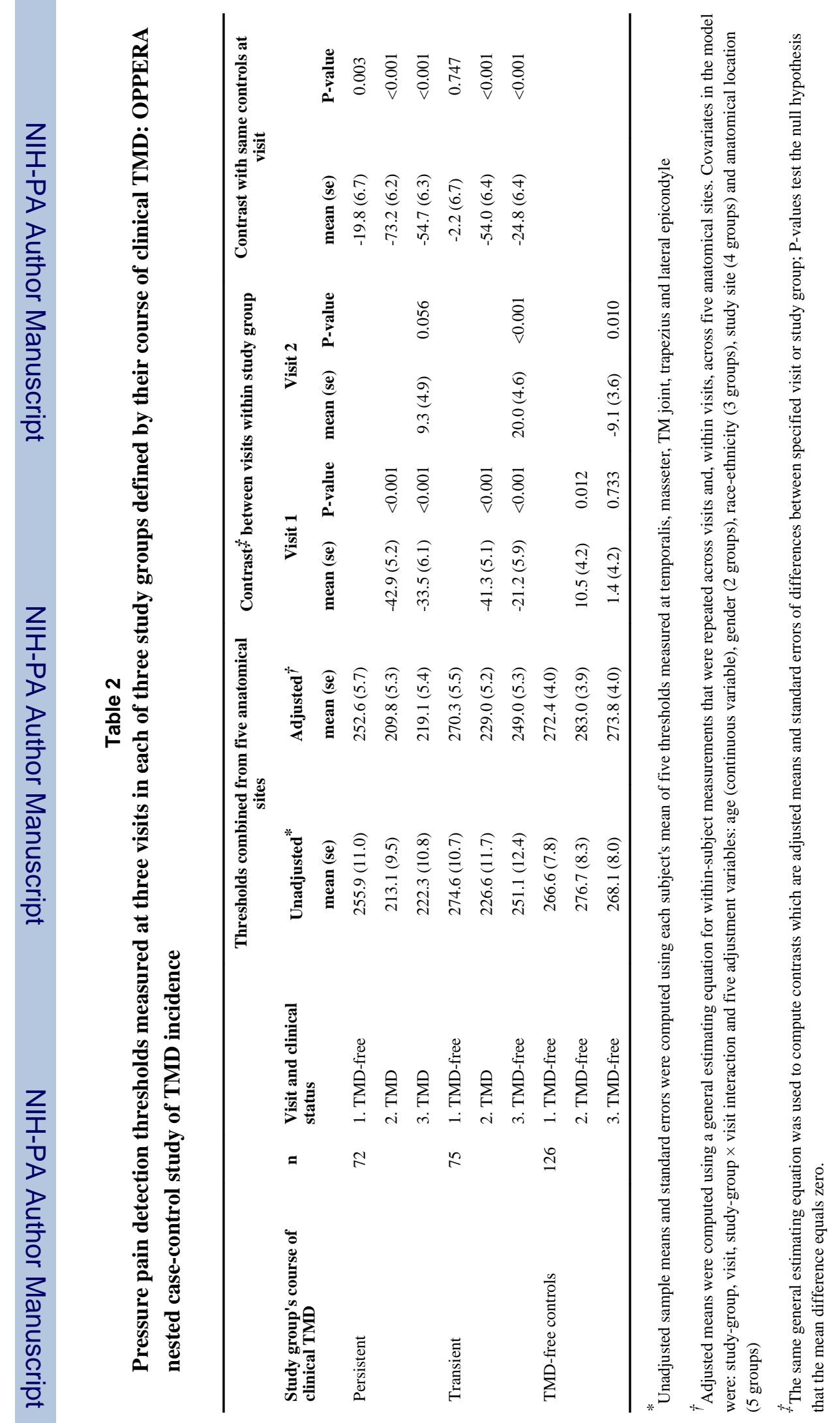

Pain. Author manuscript; available in PMC 2015 October 01. 


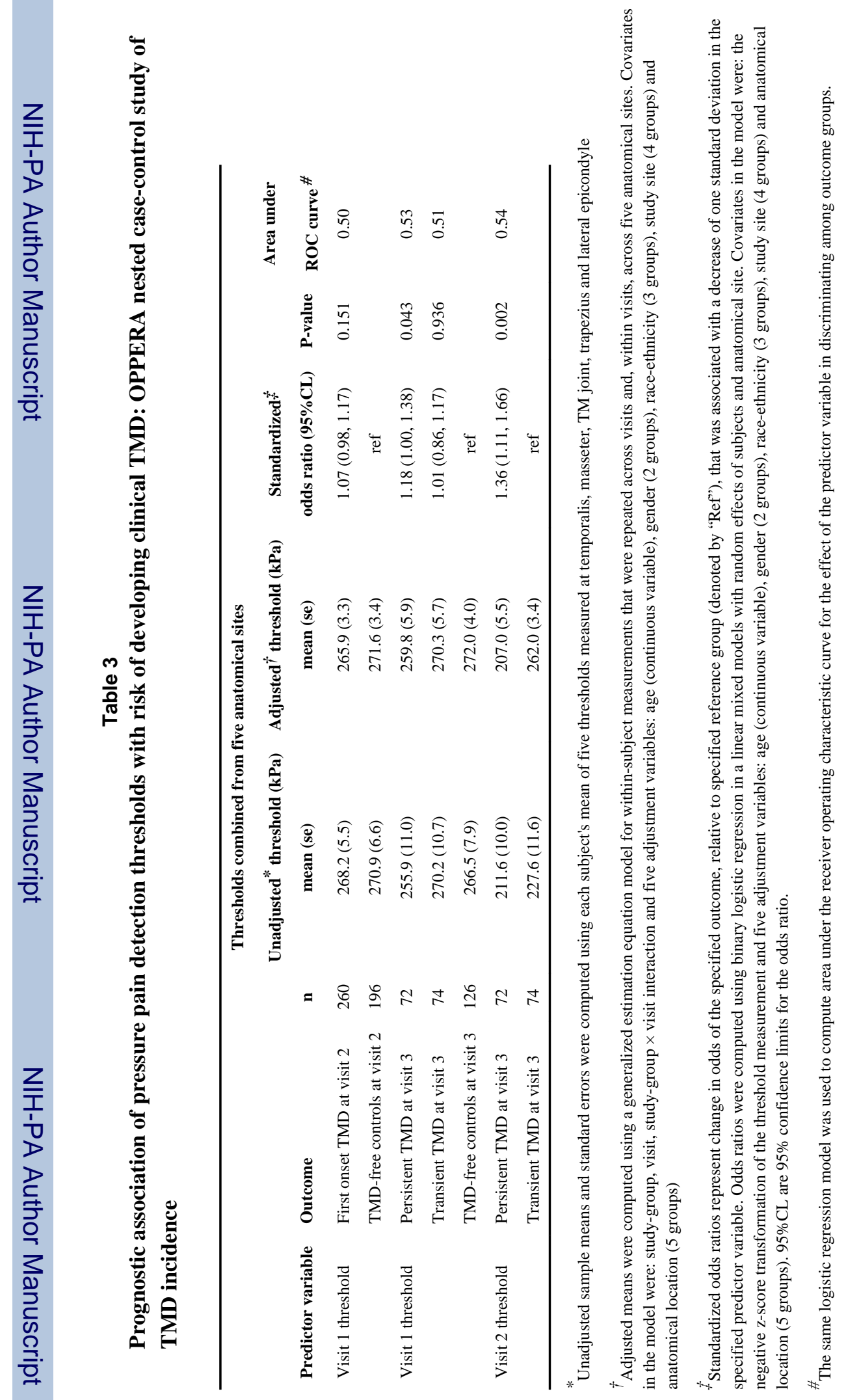

Pain. Author manuscript; available in PMC 2015 October 01. 


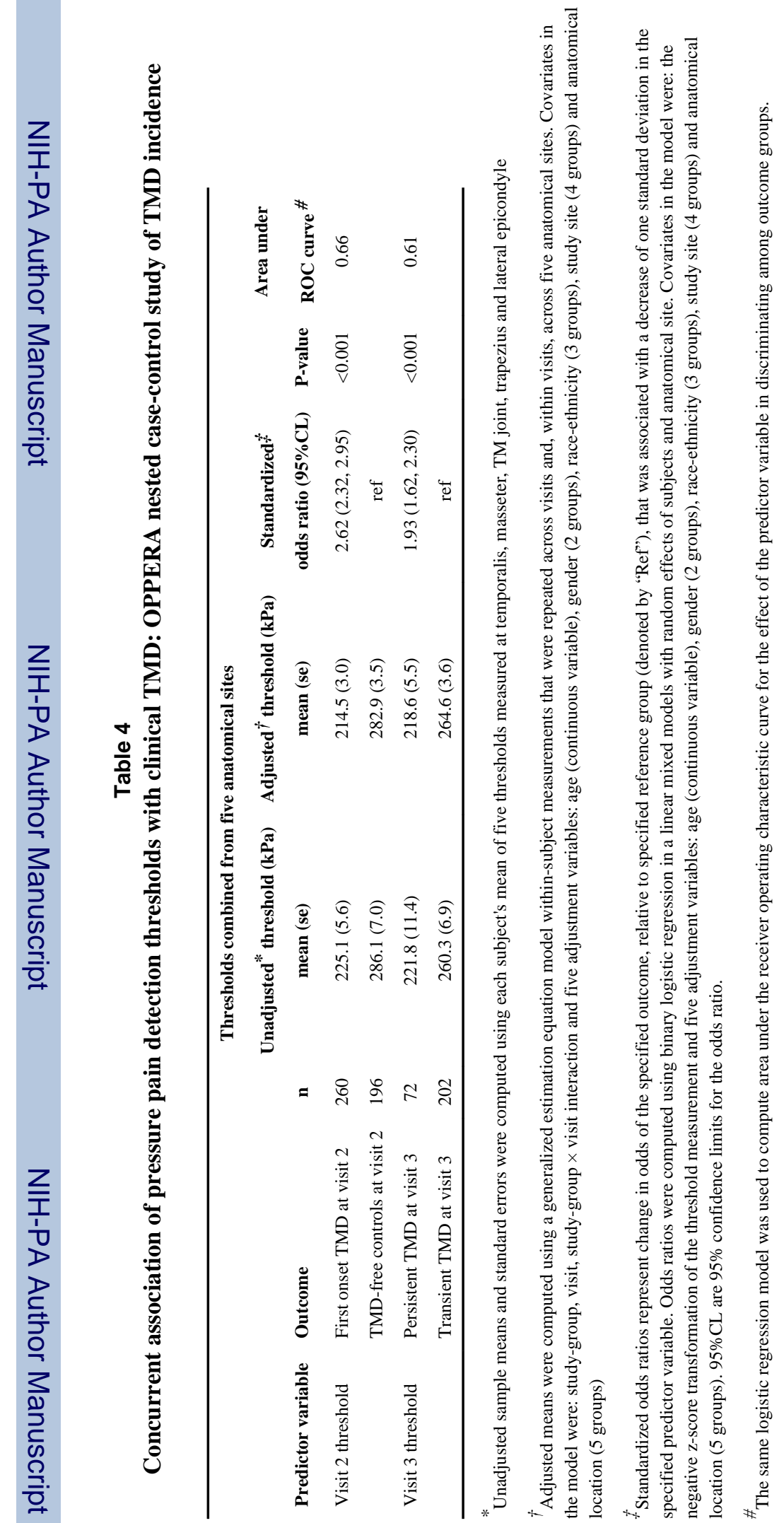

Pain. Author manuscript; available in PMC 2015 October 01. 